\title{
Overall Model of Plasticity and Failure of Metals
}

\author{
L.B. ZuEV*
}

Institute of Strength Physics and Materials Science, SB RAS, Tomsk, 634021, Russia

\begin{abstract}
Plastic flow evolution was investigated for various metals and alloys, which differed in chemical bond and crystal lattice type (BCC/FCC/HCP), structural state (single-crystal/polycrystalline) and deformation mechanisms (dislocation glide/twinning). On the base of conclusive evidence it is attempted to explain the phenomenon of plastic flow localization by invoking a fundamental principle of quantum mechanics.
\end{abstract}

PACS: 62.20.fq, 61.72.--y, 62.20.mt

\section{Introduction: autowave plastic deformation}

This paper was originated as a series of experimental study of plastic flow that was carried on for the recent two decades. The main findings of this study show that the plastic flow tends to localize at all the scale levels [1]. For a researcher in plasticity physics the macro-scale level is obviously the most convenient one since it serves to provide a unified explanation for a variety of plastic flow localization phenomena with striking regularities which are usually left unexplained. Thus it is found that (i) the plastic flow would exhibit a localization behavior from yield point to failure, (ii) each flow stage is characterized by the emergence of a specific localization pattern, (iii) the occurrence of localization patterns is explained as due to the generation of autowaves (self-excited waves), (iv) the latter waves are associated in turn with the processes of self-organization of defects.

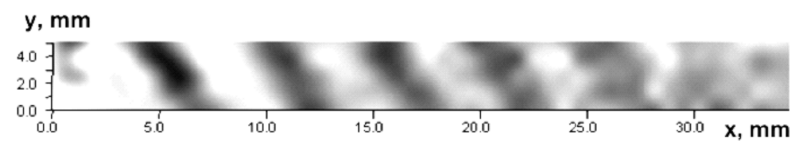

Fig. 1. Localization zones (dark bands) in the test sample.

The kinematic observations were carried on with the aid of a specially developed technique related to speckle photography [1]. A typical example of autowave patterns occurring in the deforming medium is shown in Fig 1. The propagation velocity, wavelength and frequency were defined experimentally for the autowaves in question. The values obtained differ fundamentally from those observed for elastic (ultrasound) waves [2] and plasticity waves [3], i.e. $10^{5}<V_{a w}<10^{4} \mathrm{~m} \mathrm{~s}^{-1} \ll V_{\text {sound }} \approx 3 \times$ $10^{3} \mathrm{~m} \mathrm{~s}^{-1}, \lambda \approx 10^{-2} . m \gg b \approx 5 \times 10^{-10} \mathrm{~m}$ (here $b$ is the Burgers vector of dislocations) and $10^{3}<\omega<10^{2} \mathrm{~Hz}$.

\footnotetext{
* e-mail: lbz@ispms.tsc.ru
}

\section{Dispersion relation for localized plastic flow autowaves: experimental results}

The nature of autowave processes involved in plastic flow localization is conveniently addressed using the dispersion relation $\omega(k)$ derived for the stages of easy glide and linear work hardening [4], i.e.

$$
\omega(k)=\omega_{0} \pm \alpha\left(k-k_{0}\right)^{2},
$$

where $\alpha, \omega_{0}$, and $k_{0}$ are constants which depend on the stage of work hardening and the kind of material (see Fig. 2). Note that for the stage of easy glide $\alpha<0$ and for the stage of linear work hardening $\alpha>0$.

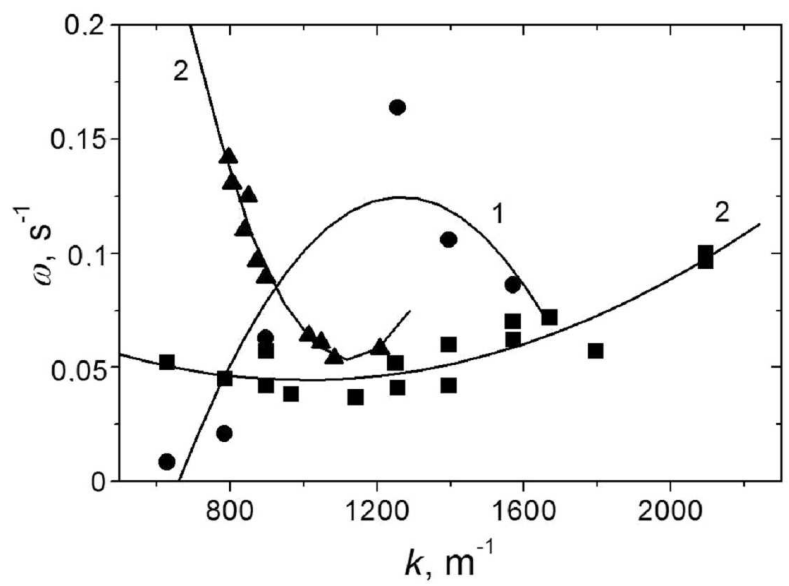

Fig. 2. Dispersion relation $\omega(k)$ obtained for easy glide (1) and linear work hardening stages (2): single-crystal $\mathrm{Cu}$, Sn and $\mathrm{Fe}(\bullet)$; single $\gamma$-Fe crystal (•), polycrystalline $\mathrm{Al}(\boldsymbol{\Delta})$.

As is seen from Fig. 3, equation (1) can be brought to canonical form by the substitution of $\omega=\omega_{0} \tilde{\omega}$ and $k=k_{0}+\tilde{k}\left(\operatorname{sgn} \alpha \alpha / \omega_{0}\right)^{-1 / 2}$ to give $\tilde{\omega}=1 \pm \tilde{k}^{2}$ (here $\tilde{\omega}$ is the dimensionless frequency, $\tilde{k}$ is the dimensionless wave number and $\operatorname{sgn} \alpha$ is a signum function of the coefficient $\alpha$. Thus we obtain the appropriate dispersion relation of quadratic form, which satisfies the Schrödinger non- 


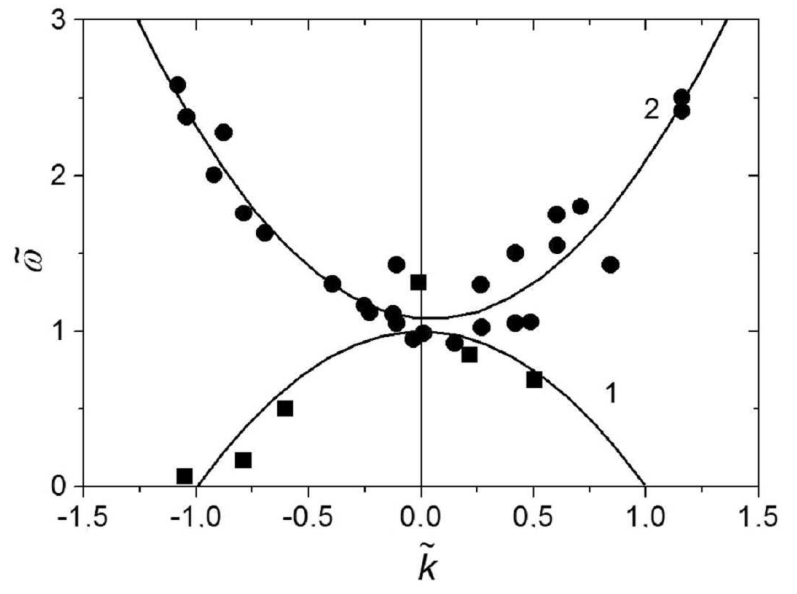

Fig. 3. A generalized relation $\tilde{\omega}(\tilde{k})$ for easy glide (1) and linear work hardening stages (2): single $\gamma$-Fe crystal and polycrystalline Al.

linear equation [5] and is descriptive of self-organization processes in nonlinear media. This is a formal proof of the fact that self-organization and plastic flow localization are closely involved processes.

\section{A correspondence between the localized plastic flow autowave and the effective mass}

The effective mass is conventionally found [6] from the dispersion law $\omega(k)$ as

$$
m_{a w}=\hbar\left(\mathrm{d}^{2} \omega / \mathrm{d} k^{2}\right)^{-1},
$$

where $\hbar=h / 2 \pi$ is the Planck constant. The effective masses obtained for single $\gamma$-Fe crystals and polycrystalline $\mathrm{Al}$ are $m_{a w}^{(\mathrm{Fe})}=0.6 \mathrm{amu}$ and $m_{a w}^{(\mathrm{Al})}=0.1 \mathrm{amu}$, respectively. Note that effective masses of the same magnitude can be calculated from the de Broglie equation [6] as

$$
m_{a w}=h / \lambda V_{a w} .
$$

Similar calculations were performed by addressing autowaves in Ref. [7]. To test the validity of this idea, $m_{a w}$ values were calculated from (3), using the autowave characteristics obtained for a number of metals and alloys [1], the results obtained are listed in the table. Apparently, the calculated values have about the same scale magnitude, i.e. $m_{\mathrm{e}} \ll m_{a w} \approx 1 \mathrm{amu}$ (here $m_{\mathrm{e}}$ is the rest mass of electron, $1 \mathrm{amu}=1.67 \times 10^{27} \mathrm{~kg}$ is atomic mass unit). The average mass calculated from (3) for the same metals is $\left\langle m_{a w}\right\rangle=(2.2 \pm 0.3) \times 10^{27} \mathrm{~kg}=1.32 \pm 0.2 \mathrm{amu}$.

TABLE

Microscopic characteristics calculated from the data on localized plastic flow autowaves.

\begin{tabular}{l|c|c|c|c|c|c|c|c|c}
\hline \hline $\begin{array}{l}\text { Metal } \\
{[\mathrm{m}]}\end{array}$ & $\begin{array}{c}\lambda \times 10^{3} \\
{\left[\mathrm{~m} \mathrm{~s}^{-1}\right]}\end{array}$ & $\begin{array}{c}V_{a w} \times 10^{5} \\
{[\mathrm{amu}]}\end{array}$ & $\begin{array}{c}m_{\text {aw }} \\
\mathrm{s} \times 10^{2} \\
{\left[\mathrm{~kg} \mathrm{~m}^{3}\right]}\end{array}$ & $\begin{array}{c}\rho \times 10^{3} \\
{\left[\mathrm{~m}^{3}\right]}\end{array}$ & $\begin{array}{c}\Omega \times 10^{27} \\
{[\mathrm{~m}]}\end{array}$ & $\begin{array}{c}d_{\Omega} \times 10^{9} \\
{[\mathrm{~m}]}\end{array}$ & $r_{\text {ion }} \times 10^{9}$ & $d_{\Omega} / r_{\text {ion }}$ \\
$\mathrm{Cu}$ & 4.5 & 8.0 & 1.1 & 1.74 & 8.9 & 0.21 & 0.059 & 0.128 & 0.46 \\
$\mathrm{Zn}$ & 4.9 & 7.6 & 1.07 & 1.66 & 7.1 & 0.25 & 0.063 & 0.071 & 0.89 \\
$\mathrm{Al}$ & 7.2 & 11 & 0.50 & 1.87 & 2.7 & 0.31 & 0.068 & 0.143 & 0.48 \\
$\mathrm{Zr}$ & 5.5 & 3.5 & 2.05 & 2.24 & 6.5 & 0.53 & 0.081 & 0.16 & 0.50 \\
$\mathrm{Ti}$ & 7.0 & 5.0 & 1.1 & 2.3 & 4.5 & 4.2 & 0.075 & 0.146 & 0.51 \\
$\mathrm{~V}$ & 4.0 & 7.0 & 1.42 & 2.81 & 6.1 & 0.33 & 0.069 & 0.135 & 0.51 \\
$\mathrm{Nb}$ & 4.5 & 4.0 & 2.21 & 2.5 & 8.6 & 0.41 & 0.074 & 0.069 & 1.08 \\
$\gamma-\mathrm{Fe}$ & 5.0 & 5.1 & 1.76 & 2.81 & 7.9 & 0.33 & 0.069 & 0.127 & 0.54 \\
$\alpha-\mathrm{Fe}$ & 4.3 & 5.2 & 1.77 & 3.0 & 7.9 & 3.75 & 0.072 & 0.127 & 0.57 \\
$\mathrm{Ni}$ & 3.5 & 6.0 & 0.89 & 3.24 & 8.9 & 0.32 & 0.068 & 0.125 & 0.54
\end{tabular}

Then the volume was calculated as $\Omega=m_{a w} / \rho$ (here $m_{a w}$ is the effective mass and $\rho$ is metal density), hence the effective size $d_{\Omega}=\Omega^{1 / 3}$. The ratios $d_{\Omega} / d_{\text {ion }}$ listed in the Table suggest that the volumes and ion radii calculated for the studied metals [8] are close in magnitude.

The average $\left\langle d_{\Omega} / r_{\text {ion }}\right\rangle$ obtained for all the studied materials is $0.51 \pm 0.1$. Normalization of the values $m_{a w}$ was performed in atomic mass units, $M_{\text {at }}$. Then the dimensionless mass was introduced, i.e. $s=m_{a w} / M_{\text {at }} \ll 1$. As is seen from Fig. 4, the value $s$ grows linearly [9] with increasing electron concentration $n$ as

$$
s=s_{0}+\kappa n=1.6 \times 10^{-2}+0.17 \times 10^{-2} n .
$$

The latter dependence is determined by the viscous drag of dislocations due to the occurrence of phonon and electron gases in metals [1].

A question I would like to address at this point is whether it is possible to propose that a certain quasiparticle exists which corresponds to the localized plastic flow autowave generated in the deforming solid. Using 


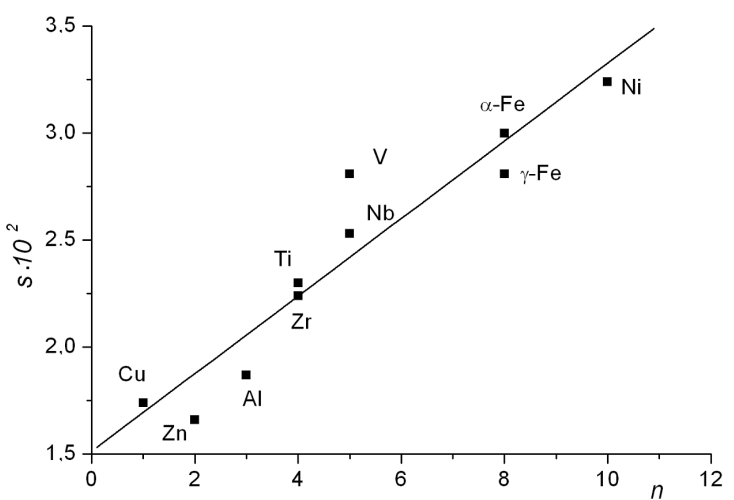

Fig. 4. Dimensionless mass $s$ as a function of electron concentration $n$; correlation coefficient $\sim 0.95$.

equation (3), the mass and the effective size of the quasiparticle can be calculated from the data listed in the table. The values obtained are $0.5 \leq m_{a w} \leq 2$ amu and $d_{\Omega} \approx r_{\text {ion. }}$. The use of this approach which has received wide application in the physics of solids [10], enables one to unambiguously relate the characteristics of the deformation macrolocalization process to those of the crystal lattice.

\section{A two-component model of plastic flow}

I would like to single out the physical aspect of the problem considered herein. This consists in the fact that a unified account is sought for two closely interrelated categories of events. Indeed, an elementary plasticity act (shear) is capable of generating an acoustic impulse, which in turn would initiate a new shear. Thus one has to account for the causal relationship between the two kinds of events occurring simultaneously in the deforming medium, i.e. the dislocation shears, which initiate the relaxation of stresses, and the acoustic emission pulses, which are responsible for the redistribution of stresses. Taking this approach, the idea of spontaneous layering [1] as applied to a system undergoing self-organization was formulated. Spontaneous layering would cause formation of two interrelated subsystems, an informative and a dynamic one. For the case of deforming medium, the choice of appropriate subsystems appears to be sufficiently simple. Thus the role of information signals, which cause relaxation of shears, is assigned to acoustic emission pulses (phonons) generated by other, similar shears. The resultant redistribution of the elastic field would initiate new shears in the dynamic dislocation subsystem.

The spatial scales of macro- and microscopic quantities under discussion differ crucially in the range $10^{6}<$ $\lambda / r_{\text {ion }}<10^{7}$. Nonetheless, it is established that the product of two macro-characteristics of autowave processes, i.e. $\lambda V_{a w}$, and the product of two microcharacteristics of the respective metal, i.e. $r_{\text {ion }} V_{\perp}$, are related linearly as $\lambda V_{a w} \approx \zeta_{\mathrm{Me}} r_{\text {ion }} V_{\perp}$,

where $V_{\perp}$ is the rate of transverse elastic waves [11]. The numerical factor differs in the range $0.52 \leq \zeta_{\mathrm{Me}} \leq 0.82$ for various metals, with the average being $\langle\zeta\rangle \approx 0.62$. The physical meaning of the latter factor might be deduced from equation (5) represented as

$$
m_{a w}=h / \lambda V_{a w} \approx \zeta_{\mathrm{Me}}^{-1} h / r_{\text {ion }} V_{\perp},
$$

where $h / r_{\text {ion }} V_{\perp} \equiv m_{\text {ph }}$ is the phonon mass. From equation (6) follows that $m_{a w} \approx 1.6 m_{\mathrm{ph}}$, i.e.

$$
1.5 m_{\mathrm{ph}} \leq m_{\text {aw }} \leq 2 m_{\mathrm{ph}} \text {. }
$$

The latter equation corresponds to the mechanism of dislocation generation due to phonon condensation [12]. Thus, a significant role in the evolution of localized plastic flow autowaves is assigned to the lattice characteristics.

The above should not be regarded as a mere formalization of the relationship between the characteristics of plastic flow localization on the one hand and the acoustic characteristics of the deforming medium on the other. A particular emphasis is placed herein on the role of the phonon subsystem in the evolution of localized plastic deformation, which involves (i) relaxation acts due to the motion of dislocations, dislocation ensembles and localized plastic flow autowaves and (ii) generation of elastic waves due to the acoustic emission, i.e. redistribution of elastic strains involving large-scale and small-scale relaxation events.

Generally, the above phenomena are studied independently. However, these might be grouped together in accordance with the concept [13] to address a system undergoing self-organization, which separates into two related subsystems, i.e. an informative and a dynamic one. Acoustic emission signals serve as deformation carriers for the deforming system. These are generated by relaxation shears, which initiates redistribution of elastic strain fields to cause new shears in the dynamic subsystem of mobile defects.

Apparently, relation (6) serves to formalize the connection between the kinetic characteristics of the above subsystems, i.e. the propagation velocity of elastic waves and the motion rate of dislocations in the vicinity of stress concentrators, respectively. In the frame of the proposed model, acoustic emission pulses would control the development of localized plastic flow, while those having sufficiently high energy would activate new plasticity events [14].

\section{Conclusion}

When considered in the context of the proposed model, the interaction between localized deformation nuclei is attributed to the exchange interaction by phonons. In this scenario it appears reasonable to assume that to the autowave of localized deformation corresponds a quasiparticle whose characteristics are determined by the autowave characteristics.

Further development of the proposed concept would enable one to group the localized plastic flow together 
with such phenomena as superfluidity and superconductivity, which are taken to be macro-scale manifestations of the deforming medium's quantum properties.

\section{References}

[1] L.B. Zuev, V.I. Danilov, S.A. Barannikova, Plastic Flow Macrolocalization Physics, Nauka Publishers, Novosibirsk 2008, (in Russian).

[2] R.E. Newnham, Properties of Materials, University Press, Oxford 2005.

[3] Basic Engeneering Plasticity, Ed. D. Rees, Buttreworth Heineman, London 2006.

[4] L.B. Zuev, S.A. Barannikova, Nat. Sci. 2, 476 (2010).

[5] A. Scott, Nonlinear Science, University Press, Oxford 2003.

[6] L.D. Landau, E.M. Lifshits, Quantum Mechanics, Pergamon Press, London 1977.
[7] L.B. Zuev, Ann. Phys. 16, 286 (2007).

[8] W.B. Pearson, The Crystal Chemistry and Physics of Metals and Alloys, Wiley, New York 1972.

[9] J. Soluom, Fundamentals of the Physics of Solids. Vol. I, Springer, Berlin 2007.

[10] J.M. Ziman, Electrons and Phonons, University Press, Oxford 2001.

[11] A. Everest, K. Pohleman, Master Handbook of Acoustic, McGraw-Hill, New York 2009.

[12] H. Umezawa, H. Matsumoto, Thermo Field Dynamics and Condensed States, North-Holland, Amsterdam 1982

[13] B.B. Kadomtsev, Physics-Uspekhi. 165, 967 (1995).

[14] L.B. Zuev, S.A. Barannikova, J. Mod. Phys. 1, 1 (2010). 